\title{
A Realization Method of Protocol Conversion Between Modbus and IEC61850*
}

\author{
Fan Zhang ${ }^{1}$, Yongli Zhu ${ }^{1}$, Chunyu Yan ${ }^{2}$, Jiangang Bi ${ }^{2}$, Haijun Xiong ${ }^{1}$, Shuai Yuan ${ }^{2}$ \\ ${ }^{1}$ School of Control and Computer Engineering, North China Electric Power University, Baoding, China \\ ${ }^{2}$ China Electric Power Research Institute, Beijing, China \\ Email: ncepuzf11@gmail.com
}

Received 2013

\begin{abstract}
In order to adapt to the construction needs of the smart grid, smart substation need to solve the problem of protocol conversion between the conventional non-standardized condition monitoring equipment and the standardized monitoring system. This paper proposed a realization method of conversion method between Modbus and IEC61850. Object-oriented technology is used for information model on Modbus. After the analysis of IEC61850 and MMS information and service model, to establish the model mapping relationship between IEC61850, MMS and Modbus based on the principle of minimum information point which is one to one correspondence. Combined with the implementation of SISCO MMS-EASE LITE software development kit programming, giving a realization method using QT programming techniques based on the mapping model. Finally, the establishment the interval controller as an example of protocol scheme verifies the correctness and the feasibility of protocol conversion method.
\end{abstract}

Keywords: Modbus; IEC 61850; Protocol Conversion; Model Mapping; Consolidated Monitoring Unit

\section{Introduction}

With the gradual popularization and application of intelligent substation, IEC61850 standard is widely used in substation automation system [1]. State Grid Corporation developed the substation equipment online monitoring system technical guidance in the process of building a strong and smart grid by 2011 [2]. It defined that condition monitoring system is structured into the process layer, the spacer layer and the station layer. The communication between the layers is used IEC61850 protocol. To achieve the goal of intelligent substation, on the one hand to develop the standard specification condition monitoring equipment to replace aging seriously equipment, on the other hand, the condition monitoring equipment which need not replace should be completed the standardized transformation to adapt to the current grid development needs. The conventional condition monitoring equipment mainly used in Modbus, IEC60870-5-103 (103), IEC60870-5-104(104), CAN bus standard as well as a large number of proprietary protocols nowadays $[3,4]$. Modbus whose structure is simple optimization and support a variety of characteristics of electrical interface and transmission medium is a protocol supported by most condition monitoring devices. Therefore, It is key

*Project Supported by the science and technology projects of State Grid Corporation (GY17201200047) to study the protocol conversion between Modbus and IEC61850 for the substation condition monitoring equipment standardization.

Model mapping and protocol conversion research between the IEC61850 standard and MMS, 103, 104 standard has being focused on nowadays. The research on the model mapping between Modbus and IEC61850 standard is less [5]. [6] gives a model mapping method between Modbus and IEC61850 using the System Configuration Description Language schema file for expanded implementation of the model, but does not give the specific engineering part of the protocol conversion module.[7] gives a CORBA-based the IEC61850 protocol conversion device. The method can be flexible and facilitate the realization of the protocol conversion, but due to the complexity of the CORBA ability, there are some limitations to realize it. IEC61850-8-1 has given the method to the implementation issues for IEC61850 which is to map the abstract information and services model to the Manufacturing Message Specification (MMS) [8]. Due to the complexity of the MMS, most of the current domestic manufacturers achieve the mapping of the communication services by SISCO MMS-EASE LITE tool package in the development of IEC61850 substation product. $[9,10]$ summarizes the information mapping correspondence between MMS and IEC61850 and gives the services mapping correspondence between ACSI and MMS 
service, but it cannot be used in the standardization of equipment condition monitoring access issues.

\section{The Information Model and Mapping}

\subsection{The information model of IEC61850 and MMS}

The actual communication functions and device has been described in an abstract way in IEC61850.It defines the five basic class model which include the Server, the logical device(LD), the logical node(LN), the data(Data), the data attribute(DA).A Server includes multiple LD, and each LD contains multiple LN, as well as each LN contains multiple Data. It can be seen that IEC61850 information model is a hierarchical and three-dimensional object-oriented structure.MMS is kind of specific communication service of IEC61850. The information Model of MMS is consistent with IEC61850. Each MMS application must contain at least one VMD object. VMD is the root in the object structure of the entire MMS, and other objects are included in the VMD object. Some types are included in the other sub-objects and become deeper sub-object.

In view of the MMS and IEC61850 model structure and its modeling method can carry out a similar analysis of the information structure of the Modbus protocol to complete the Modbus information modeling.

\subsection{Modbus and its Information Model}

GB/T 19582-2008 national standard gives two communication protocols about the Modbus Application protocol and service standards which included the Modbus serial link norms based on the TIA/EIA standard and the Modbus TCP/IP protocol based on the RFC793 and RFC791 standard [11,12]. And the Modbus serial link norms can divided into two communication modes of Modbus RTU and Modbus ASCII. Modbus RTU and Modbus TCP are mostly supported in substation condition monitoring equipment with its simple optimization feature. The Modbus parameter type is less, mainly includes coil, discrete, input registers and holding registers. The length of the first two is 1 bit, as the latter two is 2 bytes.

The Modbus data structure of the information is different from the IEC61850 and MMS which is a linear plane. The information data is defined by a corresponding Application Service Data Unit(ASDU). Figure 1 shows the ASDU structure of two types of Modbus. In order to more clearly describe it contains information, we will divide the Protocol Data Unit(PDU) to three parts which contains the data starting address(DSA), data size(DS) and data content(DC). DBA and DS here refer to the read or write the starting address and the length of the parameter. The specific parameter type is determined by the function code(FC). We can complete the information model of one ASDU by the object-oriented modeling technology easily.

\subsection{The Mapping Model between Modbus, MMS and IEC61850}

After the above analysis, the mapping from Modbus to MMS and IEC61850 is same to the flat-screen information points mapping to three-dimensional data objects. Figure 2 gives the mapping relationship between Modbus, MMS and IEC61850. The correspondence is separated by dotted lines. In the figure, the address field is the slave address of the Modbus RTU as well as the unit identifier of Modbus TCP.

- The Information Mapping

Modbus address field is used to identify a condition monitoring device, so the Modbus address field is mapped to logical device of IEC61850 and the domain model of MMS.DSA can be uniquely identifies a device measuring point, so DSA is mapped to DA of IEC61850 and the simple named variables. DS is represented the number of the registers or coils and so on, therefore it will be mapped to the basic data types of IEC61850 and that of MMS.

\begin{tabular}{|c|c|c|c|c|c|}
\hline & \multicolumn{2}{|l|}{ Modbus RTU } & \multicolumn{2}{|l|}{ Modbus TCP } & \\
\hline \multirow{9}{*}{$\begin{array}{l}\text { A } \\
\text { S } \\
D \\
U\end{array}$} & \multirow{4}{*}{\multicolumn{2}{|c|}{ Slave Address ( 1 byte) }} & $\begin{array}{c}\text { Transaction identifier } \\
\text { ( } 2 \text { bytes })\end{array}$ & \multirow{4}{*}{$\begin{array}{c}\text { M } \\
\text { B } \\
\text { A } \\
\text { P }\end{array}$} & \\
\hline & & & $\begin{array}{c}\text { Protocol identifier ( } 2 \\
\text { bytes) }\end{array}$ & & \\
\hline & & & Byte Count ( 2 bytes) & & \\
\hline & & & Unit identifier ( 1 byte) & & \\
\hline & \multicolumn{2}{|c|}{ Function Code (1 byte) } & \multicolumn{2}{|l|}{$\begin{array}{c}\text { Function Code } \\
\text { (1 byte })\end{array}$} & \\
\hline & $\begin{array}{c}\text { Data Starting } \\
\text { Address }(2 \text { bytes })\end{array}$ & \multirow{3}{*}{ 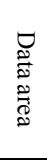 } & $\begin{array}{l}\text { Data Starting Address } \\
\text { ( } 2 \text { bytes })\end{array}$ & \multirow{3}{*}{ 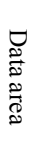 } & P \\
\hline & Data Size ( 2 bytes) & & Data Size ( 2 bytes) & & \\
\hline & Data Content & & Data Content & & \\
\hline & \multicolumn{2}{|c|}{$\begin{array}{l}\text { CRC error check code } \\
\text { ( } 2 \text { bytes) }\end{array}$} & & & \\
\hline
\end{tabular}

Figure 1. The ASDU structure of Modbus.

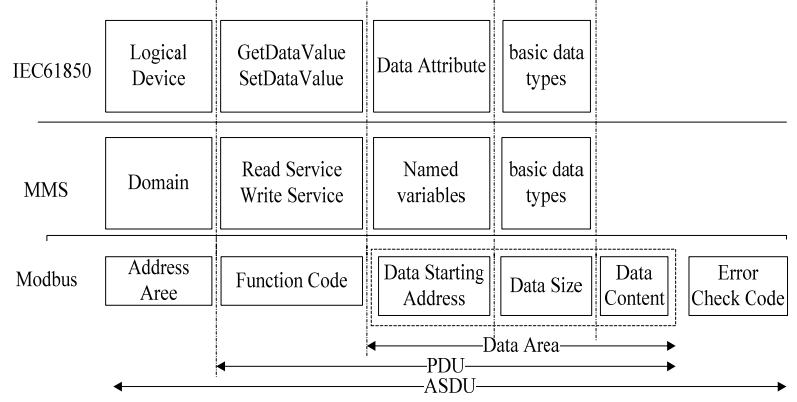

Figure 2. The mapping relationships between Modbus, MMS and IEC61850. 
- The Service Mapping

The function code specifies the different Modbus type of service, so it should be established the mapping from Modbus FC to the ACSI services or MMS services. The relationship is shown in Table $\mathbf{1 .}$

\section{The Realization of Protocol Conversion System Based on the Mapping Model}

\subsection{Design of System Architecture}

We designed the following protocol conversion mode based on the mapping model. Firstly, the point data model of Modbus map to the variable of MMS, and then to finish the mapping from MMS to ACSI. Finally, to complete the Modbus protocol conversion to IEC61850. Figure 3 is a system architecture block diagram. The substation online monitoring system Technical Guidelines require the protocol conversion module should be decorated in comprehensive monitoring unit(CMU) to support multiple protocols translation of the state devices. In order to complete the following Modbus status monitoring device communication, we need to design the modbus master model in protocol conversion module.

Table 1. The mapping table of modbus function code.

\begin{tabular}{cccc}
\hline FC & ACSI Service & $\begin{array}{c}\text { MMS } \\
\text { Service }\end{array}$ & $\begin{array}{c}\text { Functional } \\
\text { Description }\end{array}$ \\
\hline 0x01,0x02 & GetDataValues & Read & $\begin{array}{l}\text { Read the single } \\
\text { point status data }\end{array}$ \\
0x03,0x04 & GetDataValues & Read & $\begin{array}{l}\text { Read the Integers, } \\
\text { floating point data }\end{array}$ \\
$0 \times 05,0 \times 0 F$ & SetDataValues & Write & $\begin{array}{l}\text { Set the single point } \\
\text { of state control }\end{array}$ \\
& $\begin{array}{c}\text { SetDataValues } \\
\text { SetSGValues }\end{array}$ & Write & $\begin{array}{l}\text { Set the control } \\
\text { operation status }\end{array}$ \\
\hline
\end{tabular}

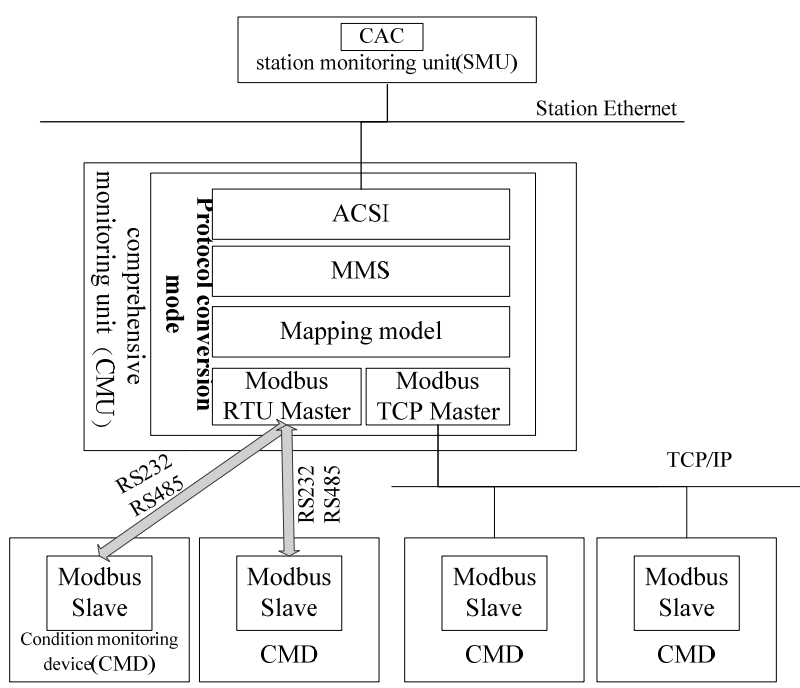

Figure 3. The system architecture block diagram.

\subsection{Realization of System Software}

- Design of the Software Architecture

QT as the software development tools is used in the system. Figure 4 is the design of software structure diagram. The system overall is design for three layers which include the top-level for MMS service layer, the middle-level for protocol conversion layer and the low-level for Modbus service layer. The top-level provide MMS services to communicate with the station monitoring unit (SMU) or the standard client of IEC61850. If the service request which get from SMU is correct, it will send the request to the middle-level. The MMS requested service data in MMS-EASE LITE is encapsulated in the structure MVLU_XX_VA_CTRL, if the service is read, XX is $\mathrm{RD}$, otherwise $\mathrm{XX}$ is WR. The low-level receive or send Modbus messages as a Modbus Master to communicate with the condition monitoring devices(CMD). The Modbus response data information from the correct response will be sent to the middle layer. The middle-level will complete the analysis of MMS service data information provided by upper and construct the corresponding Modbus request protocol frame to send to lower. And it will complete the analysis of Modbus response data from lower and construct the corresponding MMS response service to send to upper. The signals and slots mechanism of QT has good characteristics of inter-thread communication, so the information exchange of system between the three layers can utilize the mechanism to complete. Figure 6 shows the signals and slots function of the software design and identifies the relationships between the different signals and slots. The solid line represents the system receives signals and slots from the Modbus Response from CMD and the data flow. The broken line represents the association of the signals and slots after receipt of the MMS request and data flow.

- Design of Modbus class diagram

\begin{tabular}{|c|c|c|c|}
\hline $\begin{array}{c}\text { MMS } \\
\text { Response }\end{array}$ & \multicolumn{3}{|l|}{\begin{tabular}{c} 
MMS \\
Request \\
\hdashline$-j$
\end{tabular}} \\
\hline & Signal and Slot Function & Signal/Slot & Description \\
\hline & -mms_service_rd(MVLU_RD_VA_CTRL*) & SIGNAL & \multirow{4}{*}{ Top-layer } \\
\hline & -mms_service_wr(MVLU_WR_VA_CTRL*) & SIUNAL & \\
\hline & on_response_rd(MVLU_RD_VA_CTRL*) & \multirow[t]{2}{*}{ SLOT } & \\
\hline & on_response_wr(MVLU_WR_VA_CTRL*) & & \\
\hline & onWriteDataReady (QByteArray) & \multirow{3}{*}{ SIGNAL } & \multirow{6}{*}{$\begin{array}{l}\text { Middle- } \\
\text { layer }\end{array}$} \\
\hline & onRdResponse(MVLU_RD_VA_CTRL*) & & \\
\hline & onWrResponse(MVLU_WR_VA_CTRL*) & & \\
\hline & onRequestRd(MVLU_RD_VA_CTRL*) & \multirow{3}{*}{ SLOT } & \\
\hline & onRequestWr(MVLU_WR_VA_CTRL*) & & \\
\hline & -onRecieveData (QByteArray) & & \\
\hline & Edevice_data_ready (QByteArray) & SIGNAL & \multirow{2}{*}{ Low-layer } \\
\hline & -write_data_device(QByteArray) & SLOT & \\
\hline $\begin{array}{c}\text { Modbus } \\
\text { Response } \\
\end{array}$ & $\begin{array}{c}\text { Modbus } \\
\text { Request } \\
\end{array}$ & & \\
\hline
\end{tabular}

Figure 4. The design of software structure diagram. 
We complete the Modbus information modeling with the object-oriented approach. Figure 5 shows the Modbus class diagram design. The ModbusFrame class is used to describe the parameters of the Modbus ADU. Establishing ModbusServer abstract class as an abstract of the Modbus function can be inherited by different interface communication protocols for service. ModbusServerTCP and ModbusServerRTU inherited from ModbusServer what achieve the service of Modbus TCP and Modbus RTU respectively. We established two subclasses for ModbusServerRTU. The two subclasses are responsible for building and parsing of the response or request of the Modbus RTU. In addition, we create an abstract class FrameBase. FrameBase can be inherited by different subclass which is decided by function code and can complete the building or parsing of different Modbus frame.

- The process of software

Figure 6 shows the flowchart of the system software. The system can be designed two threads, one thread is used for receiving the MMS requests from SMU or IEC61850 client, and another $r$ is used for receiving the Modbus response from CMD. As a CMU should connect to multiple $\mathrm{CMD}$, we provide a thread reincarnation scanning up to four serial ports or network ports in order to ensure the overall operating efficiency of the system. It is to say we should increase the number of threads to appropriate the number of devices. In addition, taking into account the MMS communication frequency of the upper and lower Modbus communication frequency inconsistency, we establish the policy of the pipe.

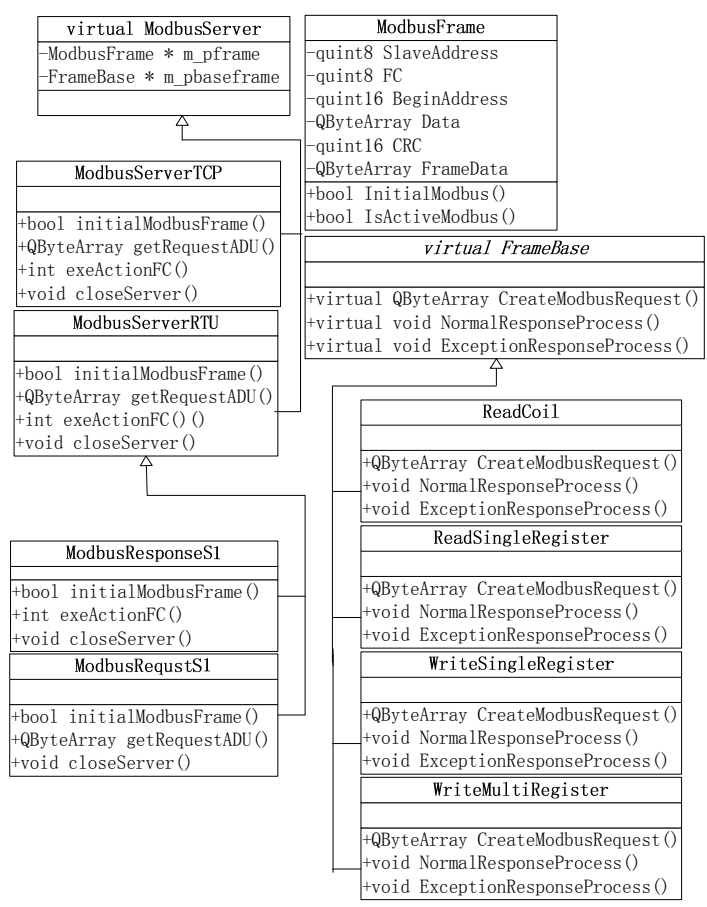

Figure 5. Modbus class diagram.

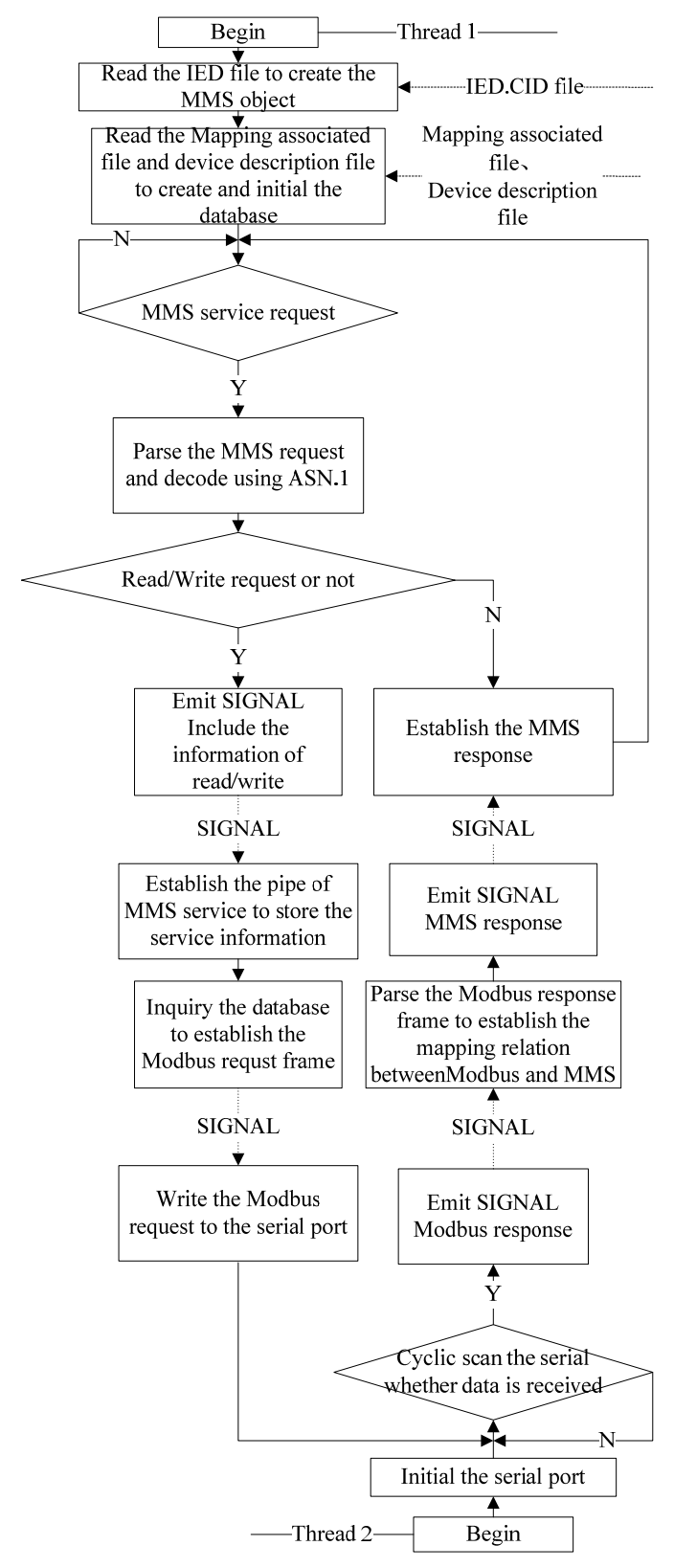

Figure 6. The flow chart of software.

System need tree files at initialization time which include the condition monitoring device description file, the device IED configuration file and the mapping associated file. The device description file is given by the production manufacturers. The file should contain the representation method of the device data information in Modbus PDU and so on. The device IED file is generated by SCL configuration tools and should follow the SCL modeling rules.The mapping associated files need manually or point table tool to carry out.In order to achieve the procedures of the system mapping relationship conveniently and efficiently,A embedded relational database which is SQLite is needed to establish three relational 
tables as the mapping model. Table 2 shows the basic information mapping table for storage Modbus informationg and MMS variable mapping relationship. Table 3 shows the writing services relationship and Table 4 shows the reading services relationship. The initialization of database is to resolve the mapping associated file which establish by manual.

Table 2. Basic information mapping table.

\begin{tabular}{ccc}
\hline Field & Type & Description \\
\hline MMSVariable & VarChar(65) & MMS variable name \\
SlaveAddress & Int & Modbus address area \\
StartAddress & Int & Modbus data starting address \\
DataSize & Int & Modbus data size \\
\hline
\end{tabular}

Table 3. The service relationship of read.

\begin{tabular}{ccc}
\hline Field & Type & Description \\
\hline MMSVariable & VarChar(65) & MMS variable name \\
FunctionCode & Int & $\begin{array}{c}\text { Modbus Function Code } \\
(\text { (0x01 0x04) }\end{array}$ \\
\hline
\end{tabular}

Table 4. The service relationship of write.

\begin{tabular}{ccc}
\hline Field & Type & Description \\
\hline MMSVariable & Varchar(65) & MMS variable name \\
FunctionCode & Int & $\begin{array}{l}\text { Modbus Function Code } \\
(0 \mathrm{x} 05,0 \mathrm{x} 06,0 \mathrm{x} 0 \mathrm{~F}, 0 \mathrm{x} 10)\end{array}$ \\
\hline
\end{tabular}

Table 5. A part of the data object of interval controller mapping relationship.

\begin{tabular}{|c|c|c|c|c|c|}
\hline \multirow{2}{*}{$\begin{array}{c}\text { MMS } \\
\text { MMS objects }\end{array}$} & \multicolumn{5}{|c|}{ Modbus } \\
\hline & $\begin{array}{l}\text { Slave } \\
\text { address }\end{array}$ & $\mathrm{FC}$ & DSA & DS & Description \\
\hline $\begin{array}{l}\text { MMXU1\$MX\$A\$phs } \\
\text { A \$cVal\$mag\$f }\end{array}$ & 01 & 04 & 0001 & 2 & $\begin{array}{l}\text { A phase } \\
\text { current }\end{array}$ \\
\hline $\begin{array}{l}\text { MMXU1\$MX\$A\$phsB } \\
\text { \$cVal\$mag\$f }\end{array}$ & 01 & 04 & 0003 & 2 & $\begin{array}{l}\text { B phase } \\
\text { current }\end{array}$ \\
\hline $\begin{array}{l}\text { MMXU1\$MX\$A\$phsC } \\
\text { \$cVal\$mag\$f }\end{array}$ & 01 & 04 & 0005 & 2 & $\begin{array}{l}\text { C phase } \\
\text { current }\end{array}$ \\
\hline $\begin{array}{l}\text { MMXU1\$MX\$PPV\$p } \\
\text { hsAB\$cVal\$mag\$f }\end{array}$ & 01 & 04 & 0007 & 2 & $\begin{array}{l}\text { AB line } \\
\text { voltage }\end{array}$ \\
\hline LLN0\$ST\$Mod\$stVal & 01 & 0406 & 0011 & 2 & $\begin{array}{l}\text { Device mode } \\
\text { of operation }\end{array}$ \\
\hline
\end{tabular}

\section{Case Study}

MMS-EASE LITE gives the corresponding IED configuration file of the interval controller E1Q1SB1. We will complete the mapping model of E1Q1SB1 by the protocol conversion method. First, MMS objects should be created according to the IED file to complete the mapping from MMS to IEC61850 standard. Then the mapping model should be established from MMS objects to Modbus information model after the analysis of MMS objects. In the example, we test a part of data objects and properties of logical Node MMXU1 and LLNO. The Table 5 shows the testing objects. Finally, we will read or write the phase current, line voltage and so on from the analog interval controller by the IEC61850 standard client.

By simulation, we can convert the ACSI or MMS service request to corresponding Modbus request correctly and return the corresponding Modbus request by the analog sub-station of Modbus. The system can parse the information data integrally and return the response of ACSI or MMS correctly. In addition, the pipeline strategy makes the condition monitoring equipment and station-side monitoring device to achieve asynchronous communication and solves the inconsistent problem of the communication frequency between MMS and Modbus. The process and results of the test results show that the system is stable and reliable.

\section{Conclusions}

The paper researches the information of MMS, IEC61850 and Modbus and their mapping relationship between their models firstly. The model can accurately describe the point information and can be more easily achieved with MMS and IEC61850 mapping. According to the Modbus information model, a protocol conversion method is given by QT technology and MMS-EASE LITE tools package then. The program not only facilitates the realization of the protocol conversion, but also provides a guideline to the private protocol which has a linear plane features mapping with IEC61850. Finally, Interval controller as an example verifies the feasibility and correctness of the established mapping model and protocol conversion program. The testing results are satisfactory.

\section{REFERENCES}

[1] S. Li, Z. L. Jia and Z. H. Ying, "Reconstruction of Non-integrated Automation Substation Based on IEC61850," Electric Power Automation Equipment, Vol. 30, No. 5, 2010, pp. 139-141.

[2] State Grid Corporation, "Online Monitoring System of Substation Equipment Technical Guidelines," Beijing, 2011. 
[3] D. W. Wang, Y. L. Zhu and Y. Wang, "Condition Monitoring and Integrating Platform for Power Transmission and Transformation Equipment Based on IEC61850," Automation of Electric Power Systems, Vol. 34, No. 13, 2010, pp. 43-48.

[4] D. W. Wang, Y. Wang and J. Di, "Design Scheme of Condition Monitoring System for Smart Substation," Automation of Electric Power Systems, Vol. 35, No. 18, 2011, pp. 51-56.

[5] J. Zhang and S. Z. Hou, "Research on IEC 61850 Sever Application Based on MMSEASE Lite," Telecommunications for Electric Power System, Vol. 32, No, 227, 2011, pp. 55-58.

[6] D. W. Wang, C. Y. Yan and J. G. Bi, "An Approach to Mapping Between Modbus and IEC61850 for Condition Monitoring Communication Gateway in Substations," Automation of Electric Power Systems, Vol. 36, No. 19, 2010, pp. 78-84.

[7] W. L. Wang, G. H. Xu and Z. H. Zhu, "Research of Protocol Gateway Device Based on CORBA Technology," Power System Protection and Control, Vol. 37, No. 7,
2009, pp. 63-66.

[8] IEC61850-8-1 Communication networks and systems in substations, Part 8-1: Specific communication service mapping(SCSM) - Mappings to MMS(ISO9606-1 and ISO9506-2) and to ISO/IEC8802-3[S].2004.

[9] K. Dong, B. Guan and W. Wang, "Research on Mapping between IEC61850 and MMS," Power System Protection and Control, Vol. 38, No. 10, 2010, pp. 92-95.

[10] S. J. Wang, S. R. Ye and L. Zhang, "Analysis of Implementing the IEC61850 Model with MMS-EASE Lite," Instrumentation Technology, No. 6, 2009, pp. 50-53.

[11] GB/T19582.1-2008 National Standard of the People's Republic of China "The Industrial Automation Network Specification Based on Modbus Part 1: Modbus Application Protocol," 2008.

[12] GB/T19582.2-2008 National Standard of the People's Republic of China "The Industrial Automation Network Specification Based on Modbus Part 2: Modbus Protocol on the Serial Link Implementation Guide,” 2008. 